\title{
La irrigación en la Bética romana: las fuentes escritas para el estudio de los humedales
}

\author{
Cañizar Palacios, J.L'‥, Fornell Muñoz, A. ${ }^{2}$, López Medina, $\mathrm{M}^{\mathrm{a}} \mathrm{J}^{3}$ \\ Universidad de Cádiz ${ }^{1}$, Universidad de Jaén ${ }^{2}$, Universidad de Almería ${ }^{3}$ \\ joseluis.palacios@uca.es, afornell@ujaen.es, jlmedina@ual.es
}

\begin{abstract}
This paper is part of the studies of the Research Project "Riparia. Conceptualization, management, exploitation and function of Wetlands and the lacustrine spaces in the Roman Baetica". Through analysis of the diverse documentary sources, particularly from the epigraphic and legal ones, it tries the conceptual definition of lacustrine and wetland spaces and its reflection in those who integrate the riverside areas of the Roman Baetica. This Roman region was a space that counted on diversity of wetlands and lacustrine areas, as much in the coast as in the interior, and had to be organized, to be regularized and to be used by the Roman power from its inception in the territory after the creation in $197 \mathrm{BC}$ of the Ulterior Baetica province, to the object of its correct exploitation and economic advantage taking into account the resources that it provided.
\end{abstract}

Keywords: Wetlands, Baetica, agriculture, irrigation, written sources

\begin{abstract}
Resumen. La presente comunicación forma parte de los estudios del Proyecto de Investigación "Riparia. Conceptualización, gestión, explotación y función de los espacios lacustres en la Bética romana" y pretende la definición conceptual, a través del análisis de las diversas fuentes documentales, particularmente de las epigráficas y jurídicas, de los espacios lacustres y humedales y su reflejo en aquellos que integran los medios ribereños de la Bética romana. Esta región romana fue un espacio que contó con diversidad de zonas humedales y lacustres, tanto en la costa como en el interior, y hubo de ser organizado, regularizado y utilizado por el poder romano desde su aparición en el territorio tras la creación en el 197 a.C. de la provincia Ulterior Baetica, al objeto de su correcta explotación y aprovechamiento económico habida cuenta de los recursos que suministraba.
\end{abstract}

Palabras clave: humedales, Baetica, agricultura, irrigación, fuentes escritas

\section{Introducción}

Este trabajo forma parte del proyecto de investigación "RIPARIA", cuyos objetivos han sido presentados con el mismo título por el Investigador Principal Lázaro G. Lagóstena Barrios en el Colloque International: Nouvelles aproches de l'Ingénierie bydraulique romaine, celebrado en Toulouse del 15 al 16 de febrero de $2013^{2}$. En él vamos a realizar una aproximación a la irrigación y los distintos espacios medioambientales que localizamos en el sur peninsular, así como a la conceptualización de los espacios lacustres y humedales en el mundo romano, y su aplicación en la Bética romana. Para ello vamos a centrarnos en el análisis de las fuentes escritas, no sin antes recordar el protagonismo que el agua alcanzó en el imperio romano.

\footnotetext{
1 "RIPARIA: conceptualización histórica y cultural, función territorial y aprovechamiento de los humedales en la Bética romana” (HAR 2012-36008, MINECO).

2 Sobre el análisis por la investigación actualmente del concepto riparia, sus usos, gestión, etc. véase Hermon y Watelet (2014) y Hermon (2010).
} 
En este sentido no cabe duda que a la autoridad romana le ocupó y preocupó siempre el correcto uso y administración del agua, recurso indispensable tanto para garantizar el suministro a los núcleos habitados como para su aprovechamiento agrícola ${ }^{3}$. Muestra de este interés por cuestiones relativas al abastecimiento, canalización y almacenamiento de las aguas lo constituye el contenido del libro VIII del De architectura de Vitrubio, en particular en sus capítulos 3 y 5 , elaborado además en un contexto histórico que viene marcado por una política encaminada a la exaltación de Roma y a la consolidación del nuevo régimen de gobierno instaurado por Augusto, expresión de lo cual serían las obras de ingeniería asociadas al abastecimiento de agua ${ }^{4}$. En la misma línea argumental cabría interpretar otras fuentes como el De aquaeductu urbis Romae, tratado de Julio Frontino, curator aquarum para el abastecimiento de la capital en el 97 d.C., y en particular la ingente cantidad de jurisprudencia referida al asunto y conservada en el Digesto, así como la presencia de articulado en la legislación municipal y la existencia de normativa tardoimperial alusiva a la tarea de la administración, gestión y uso del agua ${ }^{5}$.

Acorde con todo ello podría definirse acertadamente a los romanos como "ingenieros del agua" dada la elaboración y desarrollo de sistemas de captación, almacenaje y conducción del líquido elemento, en ocasiones altamente complejos, lo que a veces podía provocar modificaciones en el paisaje ${ }^{6}$, siendo a menudo necesario conciliar la conveniencia de la obra en cuestión con preceptos de tipo religioso ${ }^{7}$. En todo caso las tareas de captación, almacenaje y transporte del agua serían cuestiones de capital importancia, sobre todo en aquellos espacios en los que el suministro estuviese condicionado bien por la orografía del terreno bien por la misma aridez del clima o bien por ambas cosas, tal y como probablemente sucedería en determinadas áreas de la Bética. Pasemos a examinarlo.

\section{Irrigación y diversidad geográfica en la Bética}

En la descripción realizada del territorio de Hispania por Pomponio Mela en su De Chorographia durante la primera mitad del s. I d.C., se habla de la existencia de zonas caracterizadas por la penuria aquarum (Mela, De Chorographia II.86). Se trata además de una noticia que puede estimarse como fiable, no sólo por el probable nacimiento de Pomponio Mela en la Bética (exactamente en Tingentera, esto es, entre Carteia y Melaria: Mela, De Chorographia II.96), sino porque se reconoce que su obra reflejaría en su conjunto la realidad geográfica, político-social y etnográfica de la Hispania de su

\footnotetext{
${ }^{3}$ En efecto, resulta habitual en las fuentes literarias que se le conceda una elevada relevancia, tal y como reflejan por ejemplo Vitruvio en su tratado de arquitectura o Plinio el Viejo en su Historia Natural. En el primer caso puede citarse lo dicho en Vitruv. De arch. VIII.1.1 y en el segundo lo afirmado en Plin. N.H. II.63.155. Para el primero usamos la edición latina de Callebat (1973). En el segundo empleamos la edición latina en 10 volúmenes de Goold (1979).

${ }^{4}$ La construcción de una imponente obra de ingeniería, como sería un acueducto, no siempre respondería a una necesidad en el suministro de agua, participando entonces de una finalidad honorífica o incluso tal vez publicística. Al respecto véase Leveau y Paillet (1983, p. 234).

${ }^{5}$ En cuanto a la administración del agua por la autoridad romana Brunn (2012) y Bianco (2007).

${ }^{6}$ Las fuentes escritas así lo atestiguan, como por ejemplo se infiere de lo dicho en la conocida correspondencia del emperador Trajano con Plinio el Joven en ocasión del gobierno de éste último en la provincia de Bitinia, y en particular en la que se alude a un proyecto que preveía en el territorio de Nicomedia la conexión de un lago de grandes dimensiones -amplissimus lacus- (en la actualidad el lago Sabandja, en Turquía) con la línea de costa, intentando comunicar así el golfo de Ismud con el Mar Negro, proyecto que sin embargo nunca llegó a concretarse (Plin. Ep. X.41, 61 y 62).

${ }^{7}$ En particular a la capacidad para desviar el curso de los ríos y la construcción de canales sin menoscabo del cumplimiento de preceptos de índole religioso véase Moreno Herrero (2012).
} 
tiempo (Parroni, 2008). También Estrabón en su Geografía, al comentar el clima de la Península Ibérica, formula una valoración parecida ${ }^{8}$. Si ello es así, a buen seguro que debiera contarse entre las mencionadas zonas pobres en agua a la parte más oriental de la Bética, sin duda sujeta entonces, como ahora, a condiciones climáticas áridas que de modo efectivo se dejarían notar en la disponibilidad de agua?

Tanto la cita de Mela como la de Estrabón están confirmadas, para el caso del sureste peninsular, por las reconstrucciones paleoambientales. Se sabe que aquí el clima no ha experimentado grandes cambios desde la Prehistoria reciente (Pantaleón-Cano et al, 1996). Sin embargo, los ríos presentaban un caudal mayor y estable (Hernando, 1987), por lo que los recursos acuíferos eran más abundantes. En consecuencia, ha sido el paisaje el que se ha transformado debido a la acción antrópica, siendo en época romana la línea de costa distinta, pues en la desembocadura de los principales ríos del sur peninsular se formaban estuarios (Hoffmann, 1988; Arteaga et al, 1985). Además debió de existir una mayor cobertura vegetal, frente al paisaje desértico actual de algunos puntos.

En estas zonas la captación y el control del agua adquieren una importancia básica para el desarrollo de los grupos humanos que en él habitaron, pues el agua no sólo es un elemento indispensable para el consumo directo, sino que también es necesaria su presencia en ciertas actividades realizadas en época romana como son la agricultura, la minería y las labores relacionadas con la pesca, caso de las salazones de pescado ${ }^{10}$. De hecho la importancia del aprovechamiento hídrico se puede ver en la misma estructura del poblamiento, cuyos asentamientos se ubican en las márgenes de los ríos y ramblas, así como en las cercanías de las fuentes.

Pero independientemente de la gran diversidad que en la antigüedad ofrecen las condiciones medioambientales y los entornos geográficos del sur peninsular en los que se localizan los humedales, resulta de alto interés el estudio de estos espacios dispersos por el territorio bético, en particular en lo que se refiere a su utilización agrícola.

Tratamos además de un área ampliamente urbanizada y romanizada desde que conociera la organización romana a partir de su conversión en provincia en el año 197 a.C. y con otra peculiaridad añadida, esto es, la de ser un espacio que, como nos subrayan continuamente las diferentes fuentes escritas, tal vez compartiendo un tópico literario, gozaba de una importante riqueza natural ${ }^{11}$. De cualquier modo resulta

\footnotetext{
8 "Su primera parte, como ya dijimos, la occidental, es Iberia. De ésta, la mayor parte de su territorio está poco habitado: pues consiste sobre todo en montañas, bosques y llanuras de suelo pobre y ni siquiera regado de manera uniforme" (Str. III.1.2). Usamos la traducción de Gómez Espelosín (2007).

${ }^{9}$ Respecto a la situación climática y la reconstrucción paleoambiental del sureste peninsular en tiempos romanos véase Arrayás Morales - López Medina (2009); Carrilero Millán, López Medina, y García Pardo, (2004) y López Medina y Carrilero Millán (2000).

${ }^{10}$ En otros trabajos hemos analizado de manera detallada la necesidad del uso de agua en la irrigación apoyándonos en datos arqueológicos (Prieto et al, 2011; López Medina, 1998-1999). Por otro lado, debe señalarse la existencia en la línea de costa del territorio bético de espacios anegados a los que correspondería una sobresaliente incidencia en la economía de la zona, destacando en este sentido el cultivo salinero. Acerca del valor de las salinas marítimas en el entorno de la Bética véase Lagóstena et al (2010).

${ }^{11}$ P.ej. Str. III.2.4 y 6-8 o Plinio N.H. III.1.3. Ha de señalarse que en el caso de Estrabón la exageración formulada en la descripción de la Bética se inscribe en un contexto histórico y cultural en el que se ensalza el gobierno de Augusto, destacándose en este caso la bonanza de los territorios que domina Roma. Es pues una literatura al servicio del poder, tal y como además el propio geógrafo declara (Str.
} 
indudable que entre las posibilidades de explotación económica que ofrece el territorio ha de computarse en un lugar destacado su potencialidad agrícola ${ }^{12}$.

En todo caso, al tratarse de un espacio relativamente amplio, en la Bética tienen cabida, como ya hemos apuntado, distintos ecosistemas y diferentes situaciones medioambientales e hidrogeográficas, de modo que no fue uniforme, como tampoco lo es en la actualidad, la disponibilidad de agua ${ }^{13}$, siendo más urgente en unas zonas que en otras la creación de sistemas de captación (López Medina, 1998-1999) y, por ello mismo, el desarrollo de técnicas de almacenamiento que entre otras cuestiones permitieran o facilitaran la irrigación de los campos ${ }^{14}$, debiendo además precisarse en tal caso si el tipo de sistema de riego utilizado fue mayor o menor en función de la monumentalidad de la edificación hidráulica a la que se asociase (pozo, cisterna, acueducto, embalse, ...). Cuestión aparte sería la de los cultivos desarrollados, suponiéndose inicialmente un dominio de la tríada mediterránea (olivo, vid y cereal), así como el desarrollo del cultivo de hortalizas y árboles frutales, para los que resultaría esencial la técnica de regadío.

Sea como fuere, la diferente disponibilidad de agua determinó, como en general acontece en el conjunto del imperio, tanto la ocupación como el poblamiento y la organización romana de la región. En ese sentido, la densidad poblacional y el nivel de urbanización serían superiores en zonas próximas y anejas al valle del Guadalquivir e inferiores en la parte oriental de la provincia (López Medina, 2004, 1996). Junto a ello se observaría además una diferente difusión por el territorio bético de obras de ingeniería hidráulica, lo que pudiera vincularse, entre otras cuestiones, con el riego de los campos, con mayor exactitud en aquellas zonas de clima más árido, como sucedería en ciertas áreas con un régimen pluviométrico reducido.

En estrecha relación con ello ha de considerarse que determinadas regiones de la Península Ibérica, entre las que contaríamos espacios como el que aquí tratamos, mostrarían características climáticas similares a las que presentan otros puntos del imperio, caso en el Occidente romano del Norte de África, y que igualmente se significan por una amplia presencia de obras hidráulicas con vistas, entre otras cuestiones, a la irrigación de los campos ${ }^{15}$. De este modo la penuria aquarum de la que habla Pomponio Mela para algunos lugares de Hispania es también la expresión elegida por otros autores en alusión a zonas de características climáticas similares, tales como

I.1.1). En relación a la descripción formulada por Estrabón sobre la Bética véase Cruz Andreotti (2007), Alonso Núñez (1999) y Arce (1989).

En todo caso resulta mucho más frecuente la descripción tópica del conjunto de Hispania que la de la Bética. Prueba de ello es que el laus Hispaniae acaba convirtiéndose en un hábito que se perpetua a lo largo del tiempo: además de observarse en el s. I d.C. en la Historia Natural de Plinio (Plin. N.H. XXXVII.203) es posible hallarlo posteriormente en el panegírico dedicado por Pacato a Teodosio en el año 389 (Pan. Lat.II(XII).4), o en el s. VI d.C. en el preámbulo a la Historia Gothorum de Isidoro de Sevilla (Isid. Hist. Goth. Laus Hispaniae).

${ }^{12}$ Así cabe interpretar lo expresado en algunos fragmentos de la Historia Natural de Plinio (Plin. N.H. XVII.94 y XVIII.66) o de la Geografía de Estrabón (Str. III.2.3).

${ }^{13}$ En relación a la cuestión del suministro de agua en la Bética véase González Román (2011).

${ }^{14}$ Resulta indiscutible que el medio rural romano conoció el uso de técnicas de regadío, tanto en zonas áridas como en las que no lo eran. Ejemplo de ello son diversas disposiciones de la jurisprudencia, noticias literarias y material epigráfico. Sobre la reglamentación normativa del agua con vistas a su empleo en la irrigación de los campos véase Maganzani (2014).

15 Sobre la implantación de sistemas de regadío en la Península Ibérica en tiempos romanos véase Beltrán Llorís - Willi (2011). Acerca del desarrollo de sistemas de regadío en el Norte de África véase Leone (2012). 
África y Egipto ${ }^{16}$. Añádase al comentario que en concreto los estudios realizados sobre el África Proconsular indican que desde la administración romana se animaría la transformación de sus espacios anegados en tierras cultivables, potenciándose en ellos el cultivo de olivo y vid (Peyras, 2010: 113-130), caso de los llamados agri palustris y que son equiparados a agri silvestres, tal y como así se menciona en la denominada lex Manciana de época flavia. Dada la similitud climática de un lado y la existencia de zonas anegadas de otro, cabría pues pensar en un uso similar de estos espacios en el caso de la Bética.

\section{La aportación de las fuentes escritas}

Pese a la innegable relevancia de los espacios lacustres y de los humedales en el territorio de la Bética, y a que el uso de los recursos hídricos para la agricultura en época romana es cada vez más evidente ${ }^{17}$, lo cierto es que no abundan las fuentes de información escritas, siendo parcas en detalles, trátese de documentación literaria, jurídica o epigráfica, y especialmente si con técnicas de regadío se pretende relacionarlas ${ }^{18}$. La principal causa se debe al propio contexto donde se produce la irrigación, es decir, en los espacios rurales, mal recogidos en las fuentes, salvo el caso de las villae $e^{19}$. Además si tenemos en cuenta que los cultivos de regadío están asociados especialmente al policultivo y que éste es practicado principalmente en pequeñas explotaciones por campesinos (ya sea en régimen de propiedad o de arrendamiento) ${ }^{20}$, el carácter marginal de estas comunidades, su modo de vida y sus problemas en los textos escritos (literarios, epigráficos) todavía es más evidente.

De cualquier modo entendemos que deben estimarse factores como el distinto grado de difusión de la ciudad en los diferentes conventus béticos, el estatuto jurídico de los centros urbanos, su extensión, la presencia de villae y pequeñas construcciones rurales, la disponibilidad de recursos naturales y la geografía de las zona para calibrar el modo y forma en que el medio resulta adaptado a las necesidades humanas, pero también cómo las personas se adaptan al medio para su mejor y más correcta explotación.

Antes de ello convendría identificar la terminología que pueda resultarnos de interés en este estudio, que podemos considerar el inicio de una línea de investigación. Al respecto, en el s. VI d.C., en sus Etimologías, Isidoro de Sevilla define de un lado los lagos y aguas estancadas, entendiéndolas como espacios en los que el agua no fluye hacia ninguna parte (Isid. Etym. XIII.19), utilizando para ello los términos lacus y stagnum $^{21}$ y relacionándolos además con espacios donde se concentra una importante

\footnotetext{
${ }^{16}$ Parroni (1984: 341), con mención a Sall. Iug. 17.5 para África y a Curt. 4.7.6 en el caso de Egipto.

${ }^{17}$ Son cada vez más numerosos los trabajos sobre la irrigación en Hispania, así podemos mencionar para la Tarraconense, Beltrán Lloris (2011); para la Bética, Fornell Muñoz (2011). En concreto para el sureste peninsular, López Medina (1998-1999).

18 Como se ha subrayado, las fuentes vinculadas al regadío en el mundo romano centran la atención preferentemente en los alrededores de Roma (Beltrán y Willi, 2011).

${ }^{19}$ Sobre las villae véase Fornell Muñoz (2001, 2005).

${ }^{20} \mathrm{La}$ vinculación entre policultivo y pequeñas explotaciones lo hemos analizado en López Medina (2008, 2004, 2000).

${ }^{21}$ Isid. Etym. XIII.19.2: lacus est receptaculum in quo aqua retinetur nec miscetur fluctibus ... lacus stat in loco nec profuit. Et dictus lacus quasi aquae locus; XIII.19.9: lacus autem idem et stagnus, ubi inmensa aqua convenit. Nam dictus est stagnus ab eo quo illic aqua stet nec decurrat. Usamos la edición bilingüe de Oroz Reta, J. - Marcos Casquero, M.A. (2004): San Isidoro de Sevilla, Etimologías, Biblioteca de Autores Cristianos, Madrid.
} 
cantidad de agua ${ }^{22}$ y de otro lado alude a los ríos, diferenciando entonces términos como fluvius, flumen y rivus (Isid. Etym. XIII.21). En el caso de los dos primeros vocablos, a decir de los tratados de agrimensura, y en particular de los producidos durante la Antigüedad Tardía, quizá fueron conceptos útiles para distinguir las corrientes de agua privada (fluvius) de las públicas (flumen) (Peyras, 2014: 245-246). Particularmente interesante resulta en concreto la explicación realizada por Isidoro del vocablo rivus, ya que el sabio hispalense indica que las corrientes de agua que reciben este nombre "son llamadas así porque son derivados para regar, es decir, para conducir sus aguas por los campos, pues "regar" es "conducir el agua", interpretación que de otro lado vienen a confirmar diferentes pasajes de la literatura latina ${ }^{23}$. En suma, es evidente, terminología aparte, $1^{\circ}$ ) que hablamos de la existencia en el medio rural romano de lugares de almacenamiento de agua, trátese de tipo natural o creados por la mano del hombre, y desde los cuales es posible derivarla, entre otros fines, para el regadío de los campos, y $2^{\circ}$ ) que este planteamiento general sería sin discusión aplicable al marco de la Bética, territorio en el que sería sobresaliente la presencia de zonas anegadas susceptibles de cumplir este cometido ${ }^{24}$.

Si concretamos, entre las fuentes de tipo literario que nos transmiten información sobre los espacios citados destacan por encima de todas, habida cuenta de la profusión de vocablos ligados a estos lugares y de su relación con la explotación agrícola, en primer lugar los tratados de agronomía, particularmente el De res rustica de Columela, que ofrecería como principal virtud la de tratarse de una de las pocas fuentes documentales escritas que pudieran relacionarse directamente con el espacio bético, y que analizaremos más adelante.

Igualmente, la terminología vinculada al territorio rural en el que destaque la presencia de espacios lacustres es notable en los tratados de agrimensura ${ }^{25}$, como reflejan en este sentido varios de los compilados en el denominado Corpus Agrimensorum Romanorum. En ellos particularmente interesante sería la alusión a distintas señalizaciones naturales que podían servir como referencia en la delimitación del espacio, y entre las que se contarían zonas anegadas, utilidad a la que sumarían su evidente función como fuentes de aprovisionamiento de agua.

De cualquier modo, como advertíamos al inicio, ninguna de las fuentes de información apuntadas hace mención de modo preciso y concreto a la Bética romana, tratándose en todos los casos de noticias y documentación genéricas. Más ligada a la cuestión de la

\footnotetext{
${ }^{22}$ En sentido similar los habría interpretado en su comentario a La Eneida de Virgilio el gramático de fines del s. IV d.C. Mario Servio Honorato: Serv. comm. in Verg. Aen. I.126 y VIII.74.

${ }^{23}$ Es el caso de Las Geórgicas de Virgilio: "¿Qué decir de aquél que, esparcida la semilla, pone mano en el terreno y allana los montones de la seca arena y después lleva el agua a los sembrados en dóciles corrientes -rivi- y, cuando el campo agostado aridece al secarse las hierbas, he aquí que hace saltar la onda de la escarpada cima a través de pendiente sendero?" (Verg. Georg. I.105-110). Usamos la traducción de Recio García, T.A. (2000): Virgilio, Geórgicas, Biblioteca Clásica Gredos, Madrid, p.68.

${ }^{24}$ Ningún tipo de fuente, laguna o lago de la Bética en particular, y de Hispania en general, resulta mencionado en una especie de diccionario que pretendería compilar en orden alfabético los nombrados por varios autores clásicos como Virgilio o Lucano. En concreto se trata de una obra fechada en los ss. IV-V d.C. que responde al título de De fluminibus fontibus lacubus nemoribus paludibus montibus, gentibus per litteras y que se atribuye a un desconocido autor de nombre Vibius Sequester. Acerca de esta obra véase Perotti (2004).

${ }^{25}$ En relación al agua cabe diferenciar en este tipo de literatura la realizada por agrimensores al servicio del estado, y que acometen las posibles controversias jurídicas que pudieran surgir en relación al denominado modus fluminii, de la elaborada a partir de fines del s. IV d.C., vinculada al control de los cursos de agua y de las zonas húmedas. Al respecto Peyras (2014).
} 
irrigación de los campos en el territorio bético pudieran estar en cambio algunos episodios transmitidos en sus obras por Plinio el Viejo y Marco Juniano Justino (Plin. N.H. XIV.71; Just. XIV.1.4-7) ${ }^{26}$ o anteriormente por Estrabón (Str. III.2.1 y 3) y que vendrían a confirmar el uso de la técnica del regadío en el marco de la Bética.

En el caso de la información derivada de fuentes epigráficas e inscripciones nos encontramos también con limitaciones, ya que nuevamente son escasas las alusivas al territorio estudiado, de modo que una vez más es necesario recurrir a las que pueden localizarse en otros rincones del imperio ${ }^{27}$. Otro tanto cabe señalar en relación a las fuentes de tipo jurídico. En este sentido apenas se relacionaría con el ámbito bético las conocidas rúbricas 19 y 82 de la lex Imitana y algunos capítulos de la lex Ursonensis, caso de los 77, 79, 99, 100 y 104, que mostrarían el deseo e interés de la autoridad municipal por regular el uso y captación del agua ${ }^{28}$.

Detallada a rasgos generales la cuestión, pasemos al análisis de fuentes concretas, desde las literarias a las jurídicas pasando por las epigráficas.

\subsection{Los textos literarios}

Como adelantábamos en líneas anteriores, Columela constituye la fuente escrita que podemos relacionar más claramente con el espacio bético, teniendo en cuenta tanto su condición de gaditano ${ }^{29}$ como la posibilidad de que poseyera propiedades inmobiliarias en el sur peninsular, así puede interpretarse de lo dicho por él mismo en Col. r.r. III.3.3.: Nam illa videntur prodigialiter in nostris Ceretanis accidisse... ${ }^{30}$. No obstante, su excepcionalidad como fuente no solo reside en este hecho, pues la información aportada por el agrónomo alude principalmente a sistemas de regadío vinculados no a núcleos urbanos sino a las villae, es decir, a unidades de explotación agropecuaria ligadas a la gran o mediana propiedad ${ }^{31}$ y en los que desempeñarían un rol importante las zonas anegadas, tal como se sugiere en diferentes pasajes de su tratado de agricultura que a continuación pasaremos a comentar.

\footnotetext{
${ }^{26}$ Sobre las evidencias literarias en las citadas fuentes alusivas a la cuestión del posible uso de sistemas de regadío en el ámbito bético véase Beltrán Llorís y Willi (2011: 13-14).

27 Para el caso de la Bética no disponemos de documentos como la lex rivi Hiberiensis, referente al entorno de Caesaragusta, o la Tabula de Lamasba (CIL VIII 4440), alusiva al Norte de África, y que sugieran el modo de organización de la comunidad de regantes. Sobre el primer documento epigráfico véase Beltrán Llorís (2006). En cuanto al segundo véase Shaw (1982).

${ }^{28}$ En relación a los diferentes tipos de agua que pudieran ser utilizadas, y en particular a la distinción de lugares diversos de aprovisionamiento mencionados en la legislación romana, véase Rodríguez Neila, (1988).

${ }^{29}$ Como se explicita en varios pasajes de su obra: Col. r.r. VIII.16.9 y X. 185. Por otro lado, las frecuentes referencias a la Bética, de la que conserva vivencias profundas, parece indicar que debió vivir en ella un tiempo antes de marchar a Roma, probablemente hasta su juventud. Esto es deducible también por las referencias que hace a su tío Marco Columela, siempre para ensalzarlo como agricultor y ganadero entendido, lo que lleva a pensar que vivió años cerca de él adquiriendo una buena formación que luego perfeccionaría con su dilatada experiencia personal. Holgado Redondo (1988).

${ }^{30}$ Holgado Redondo (1988) y Tovar (1975), identifican Ceret con Jerez de la Frontera, Cádiz.

31 Debido a la naturaleza de nuestras fuentes, la información disponible no ilumina por igual todos los sistemas de riego. La investigación ha concedido una atención preferente a la interpretación de los restos materiales de las obras hidráulicas, que constituyen los principales testimonios de irrigación, privilegiando de este modo los situados en los alrededores de las ciudades.
} 
En la obra columeliana son así frecuentes las referencias, implícitas y explicitas, al regadío, especialmente en los libros II, $\mathrm{X}$ y $\mathrm{XI}^{32}$, al igual que existe una profusión de vocablos ligados a corrientes de agua y a zonas anegadas naturales que pueden formar parte del entorno o medio natural de la hacienda agrícola, aunque fuera del área de cultivo y del lugar donde se levanta la casa. En este sentido, es especialmente ilustrativo el pasaje donde se alude a la cría de aves acuáticas, y en concreto a los gansos (Col. r.r. VIII.13.2-3), donde se indica la conveniencia de que exista en la finca un río o lago, así como hierba abundante, precisando que en cambio no debe haber en sus inmediaciones cereales sembrados (sicubi vero flumen aut lacus est herbaeque copia neque nimis iuxta satae fruges, id quoque genus nutriendum est) ${ }^{33}$.

Los ríos (flumen) y arroyos (rivus) no solo contribuyen a moderar los calores estivales y amenizar el paisaje (Col. r.r. I.5.4), sino que también pueden suministrar agua a los prados y huertos, y a la propia casa (Tum rivos decurrentes in prata et hortos et salicta, villaeque aquas salientes demittant $)^{34}$. De esto se infiere que la relación entre ríos y arroyos e irrigación parece clara. Sin embargo, no ocurre lo mismo con las zonas anegadas, sean lagos (lacus), lagunas o pantanos (palus), pues lo único que se desprende del texto es que estos espacios, que se podían encontrar en las inmediaciones de la granja, pudieron ser aprovechados como abrevaderos para el ganado, así como lugar de caza de aves acuáticas y de pesca ${ }^{35}$.

Los canales y redes de irrigación de los medios rurales resultan difíciles de identificar debido a la su naturaleza deleznable y frágil conservación. No obstante, en ocasiones excepcionales la arqueología permite su constatación. Tal es el caso de los territorios que circundan Jaén, la Aurgi iberromana, donde los intensos trabajos arqueológicos desarrollados a partir de 1995 en Marroquíes Bajos han permitido reconstruir el modelo de ocupación que surge desde el s. II a.C. en el contexto de la victoria romana sobre los aliados cartagineses y los consecuentes reajustes en las relaciones territoriales entre los oppida indígenas. En este momento se constata una ocupación intensa en las cuencas del arroyo de la Magdalena, el arroyo El Valle y otros cauces de agua dulce, para la producción de regadío. Esto ha quedado claramente probado gracias a la excavación de los sistemas de cultivo tradicionales que han dejado en numerosos puntos de esta zona sus huellas. Estas se refieren fundamentalmente a los sistemas de distribución y almacenamiento de aguas, grandes balsas, canales y acequias que se extienden a lo largo de cientos de metros por Marroquíes Bajos y la depresión de la Magdalena. Estas tempranas explotaciones rurales de carácter campesino vinculadas al regadío se mantendrán vigentes hasta época augústea ${ }^{36}$.

Aunque los cultivos propios de las regiones meridionales hispanas eran de secano, especialmente la tríada mediterránea (vid, olivo y cereal), mediante el riego resultaría posible mejorar su rendimiento y también cultivar plantas con una mayor exigencia hídrica, como los frutales y las hortalizas (coles, espárragos, alcachofas, ciruelos, lechugas, etc.). Esto es puesto de manifiesto no solo en Columela (r.r. X.110-120; XI.3.14 y 28), sino también en Plinio (N.H. XV.42; XIX. 139 y 141) e Isidoro (Etym. XVII.10.11). Pero es de destacar en concreto la cita de nuestro agrónomo al respecto de

\footnotetext{
${ }^{32}$ Col. r.r. II.9.26; II.16.3-5; II.17.5; X. 140-145; XI.3.8-11.

${ }^{33}$ Seguimos para el original latino la edición de Rodgers, R.H., Ed., (2010): Columellae Res Rustica-Incerti Auctoris Liber de Arboribus, Oxford Classical Texts, Oxford University Press.

${ }^{34}$ Col. r.r. I.2.4.

${ }^{35}$ Así se desprende de los siguientes pasajes: Col. r.r. VI.22.2; VI.27.2; VII.9.6; VIII.13.2-3; VIII.16.1-2.

36 Véase Zafra Sánchez (1997), Serrano Peña (2004) y Serrano Peña et al. (2002).
} 
las lechugas (Col. r.r. X.185; XI.3.26), pues menciona una variedad gaditana (quae deinde candida est et crispissimi folii, ut in provincia Baetica est finibus Gaditani municipii, mense Martio recte pangitur), constituyendo la más clara evidencia literaria de la existencia del regadío en la Bética.

En lo que se refiere a la agrimensura, hablamos de un tipo de literatura de elevado carácter técnico y aplicación universal no hallando en este tipo de fuentes detalles ni alusiones a las características concretas de los territorios que se pretende organizar, no entrándose en valoraciones sobre su bonanza, posibilidades de explotación, inconvenientes, etc. Sin embargo, sí es posible extraer consideraciones generales que sirvieran como pautas de actuación, siendo desde este punto de vista aplicables al espacio de la Bética.

Recuérdese que en la definición y extensión del territorium que quedaba sujeto a la jurisdicción de una civitas romana resultaba clave la delimitación del espacio, distinguiéndose de modo amplio el urbano del rural y, en este último de forma particular, diferentes hábitats y parcelas, lo que se traduce en una red de categorías de tierras que en la práctica conformarían fronteras internas. Como apuntábamos anteriormente, en este sentido sería de gran utilidad la referencia que ofrecían elementos del medioambiente y del entorno natural pero también aquellos otros que pudieran deberse a la acción humana (por ejemplo acequias). Entre estos referentes naturales han de contarse, además de lugares como los bosques o las montañas, las zonas anegadas (trátese de lagunas, lagos, pantanos o estanques) y las corrientes de agua (trátese de ríos, torrentes o arroyos) $)^{37}$, espacios que serían relevantes para el aprovisionamiento de agua y por extensión para el riego del campo.

Por lo demás esta función de demarcación que ofrecen los espacios definidos por la presencia del agua resulta ampliamente atestiguada y conocida, por lo que no sorprende que los agrimensores usualmente los tengan presentes, señalándolos de modo preciso en un elenco de límites territoriales ${ }^{38}$.

Desde un punto de vista meramente económico, que es el que aquí más nos interesa, cabe señalar que la presencia de zonas anegadas junto a otros espacios como el bosque ${ }^{39}$ y el destinado al ganado, formaron parte del conjunto de zonas incultas que se localizaban en el paisaje agrario romano asignado a una civitas en el momento de su establecimiento, jugando no obstante un significativo papel como complemento de la economía de las gentes del lugar, siendo clave su aprovechamiento en la supervivencia

\footnotetext{
${ }^{37}$ Ejemplo de ello, en el caso de la Bética, sería el denominado Bronce de Lacimurga, fechado a fines del s. I a.C., y donde se percibe la importancia en este caso de la línea natural que demarcaría el río Guadiana para la organización territorial entre las provincias romanas de la Bética y la Lusitania. Al respecto véase Sáez Fernández (1990).

38 Así lo muestran, entre otros, distintos escritos y pasajes de Higino o Sículo Flaco, en particular en sus respectivos De condicionibus agrorum, así como otros fragmentos compilados en el Corpus Agrimensorum Romanorum (p. ej. L. 342-343, 347-348 o 359-366). Seguimos en la identificación de las obras de los agrimensores latinos la edición de Campbell, B., Ed., (2000): The Writings of the Roman Land Surveyors, Journal of Roman Studies Monograph no.9, London. Apúntese en todo caso que el fenómeno resulta aún más sobresaliente en el de los fechados en tiempos tardoantiguos, momento en el que parece incrementarse la preocupación por el control de los cursos de agua y de las zonas húmedas. En relación con ello véase Peyras, J. (2014, 1995: 199-200).

${ }^{39}$ En relación al valor económico del bosque véase Giardina, A. (1981): Allevamento ed economia della selva in Italia meridionale: trasformazione e continuità, en Società romana e produžione schiavistica I. L'Italia: insediamenti e forme econocomiche, Bari, pp. 87-113.
} 
de parte de la población, de ahí que sean tenidos en cuenta a la hora del reparto y organización de la tierra.

Así, una vez concretada una fundación colonial romana, entre las categorías de tierra que los agrimensores establecen para su división, y en concreto en las que se vincularían a los loca publica agrestia, sobresale aquella que recibe la denominación de pascua et silva, con la que muy probablemente deba relacionarse la explotación de las zonas anegadas, ya que como anticipábamos anteriormente integraba la parte inculta dentro del territorium de una civitas $^{40}$. El técnico agrimensor prevé pues como recurso necesario para el sostén económico de una comunidad, y como necesario complemento a su economía, la existencia de una zona destinada de un lado a la caza y la obtención de madera, y de otro al alimento y cría del ganado, y junto a ello espacios de aprovisionamiento de agua, algunos de los cuales, aunque no sean acreedores de un tipo concreto de categoría jurídica de tierra, bien pudieran vincularse a estos mismos lugares de caza y pesca. Acorde con ello se observa, que en el momento de constitución del territorio de una civitas, estos preceptos, que de forma general indican los agrimensores, vienen a coincidir con lo señalado por los agrónomos en relación a las características ideales que debe poseer una finca, aconsejando que comprenda, junto a una zona de tierras cultivables, otra de espacios silvestres ${ }^{41}$.

Cabe además establecer la relación entre zonas boscosas y zonas anegadas a la vista de lo expresado en otras fuentes literarias. Es el caso de Marcial, quien en dos epigramas consagrados al elogio de Hispania la formula claramente, alabando las posibilidades de caza y pesca que se ofrece en los bosques (Mart. Epigr. I.49 y IV.55) ${ }^{42}$, ámbito con el que asimismo es obvia la conexión en otro epigrama en el que se elogia la vida campestre (Mart. Epigr. I.55) ${ }^{43}$. Un segundo aspecto que refuerza la impresión de la similitud de trato entre los espacios boscosos y las zonas anegadas como lagos y lagunas es su posible consideración sagrada. Así cabe interpretar lo dicho en algunos otros epigramas del mismo autor ${ }^{44}$.

Todo lo dicho se completa con la concepción que se formula de estas zonas, esto es, su faceta como lugar de ocio y esparcimiento ${ }^{45}$. Hablamos pues de una representación positiva que asemeja zonas anegadas como los lagos con lo que en la literatura latina, y más concretamente en la poesía augústea, se entendería como un locus amoenus, participando entonces de las características de un topos literario que se aplicaría de modo general a espacios con presencia de agua (McMaster, 2014). Pero no menos cierto es

\footnotetext{
${ }^{40}$ Isidoro de Sevilla, al definir en sus Etimologías las zonas incultas, caso de los desiertos, los caracteriza como lugares en los que no se siembra, incluyendo en ellos a silvae et montes y en contraposición a los espacios productivos: ... ut sont loca silvarum et montium, contraria uberrimarum terrarum, quae sunt uberrimae glebae (Isid. Etym. XIV.8.31).

${ }^{41}$ Por ejemplo, Col. r.r. I.2.

${ }^{42} \mathrm{Su}$ condición de espacio de pesca se relaciona igualmente en otros epigramas, p.ej. Mart., Epigr. IV.30 donde se advierte a un pescador que no pesque en el lago de Bayas dada su consideración sagrada.

43 “ ¿Es capaz alguien de cultivar los fríos mosaicos de mármol / espartano y llevar estúpidamente el mañanero ¡buenos días!, / si tiene la posibilidad, feliz con los despojos del bosque, / del campo, de desplegar ante el hogar redes llenas / y llevar un pez brincando en trémula caña ...?”. Seguimos la traducción de Ramírez de Verger, A. (2001): Marcial, Epigramas (vol. I), Biblioteca Clásica Gredos, Madrid, p. 52.

${ }^{44}$ Mart. Epigr. IV.30 y IX.58.

45 P.ej. Mart. Epigr. III.20 donde se alude a un paseo en barca por la laguna Lucrina: ... Lucrino nauculatur in stagno?; o IV.22, donde una tal Cleopatra se sumerge en las limpias aguas de un lago: ... merserat in nitidos se Cleopatra lacus. En cuanto a la interpretación de este epigrama se ha relacionado la mención que al agua se formula con una función simbólica. Al respecto véase Moreno Soldevila (2003).
} 
que al mismo tiempo resulta habitual la expresión de una negativa valoración de lagunas y otros espacios anegados. Desde este punto de vista se extiende la opinión que destaca su insalubridad y falta de higiene. Así lo expresa el mismo Marcial en la crítica que realiza a una tal Basa a la que acusa de maloliente, comparándola entonces con el olor que desprenden las lagunas (Mart., Epigr. IV.4: Quod siccae redolet palus lacunae ...). Y lo mismo cabe decir de los estanques de agua salada, de los que de nuevo se afirma que desprenden malos olores (Mart. Epigr. XII.32: Odor inpudicus urcei fatebatur / Qualis marinae vix sit aura piscinae ${ }^{46}$. Juicios similares pueden observarse también en el De re rustica de Columela ${ }^{47}$. Cabe concluir pues que esta doble y contradictoria estimación que de los humedales puede hallarse en los escritores latinos no sería sino reflejo de determinados usos literarios. Lo que en todo caso resulta indiscutible es que la valoración negativa que puede hallarse de las zonas anegadas contrasta en la práctica con su interesante aprovechamiento y utilidad económica, circunstancia que los equipararía a los espacios boscosos, reforzándose pues la asociación que puede formalizarse entre las zonas de bosque y los distintos tipos de humedales (lagos, lagunas, estanques o pantanos). Y no cabe duda que en la Bética, dada la difusión de estos espacios por su paisaje, resultarían claves en la prosperidad económica de ciertas zonas.

Por lo demás, al margen de pascua et silva, otras categorías de tierra que pudieran estar relacionadas con los espacios anegados serían, dentro de los loca publica agrestia, las zonas pantanosas que no se considerasen aptas para la agricultura y que pudieran integrar el espacio de tierras denominado por los agrimensores como subsiciva, a su vez relacionado con el ager divisus et adsignatus (Castillo, 1996). E igualmente, entre los loca publica agrestia, las zonas anegadas pudieran estar en relación con la superficie estimada como locus relictus, esto es, la tierra que queda al margen del reparto y asignación, y en la que en ocasiones se originaban zonas anegadas, que lo serían únicamente de forma temporal, secándose en función de la época del año ${ }^{48}$.

De cualquier modo, trasladar a la Bética esta caracterización genérica que de las zonas anegadas y los humedales hallamos en los tratados de agrimensura no es tarea sencilla. Nos topamos de entrada con una primera dificultad, compartida además por el conjunto del territorio romano: la de establecer la exacta definición de la extensión del territorio sujeto a la jurisdicción de una ciudad romana, dada no sólo la ausencia de reglamentación al respecto sino de relación alguna entre, por ejemplo, el estatuto jurídico de ciudad y el tamaño del territorio. En todo caso en su delimitación debe estimarse como factor central la consideración de la geografía de la zona, pudiendo así confluir diferentes ecosistemas en un único territorio al objeto de garantizar no sólo una adecuada explotación sino la misma supervivencia de sus habitantes, contemplándose para ello la disponibilidad de recursos naturales, en especial de agua. Y en buena lid tal debió ser el proceder de los agrimensores en particular en aquellos espacios de la provincia Bética más escasos en recursos hídricos. De hecho el tamaño del territorio de cada una de las civitates del sureste peninsular era mayor que en otras zonas de la

\footnotetext{
46 Pese a todo la mano del hombre podía modificar esta situación. Así Isidoro de Sevilla relata en sus Etimologías que la naturaleza inicial del lago Averno fue insalubre hasta la intervención humana en tiempos de Augusto, de modo que "convirtió estos pestilentes lugares en amenos parajes" (Isid. Etym. XIII.19.8: ... expestilentibus amoena reddidit loca).

47 En concreto, así se expresa al considerar la diferente calidad del agua, señalando que la que se encuentra estancada es pestilente: pestilens, quae in palude semper consistit (Col. r.r. I.5).

48 A ello se refiere en particular Virgilio: postquam exusta palus terraeque ardore debiscunt ... (Verg. Georg. III.432).
} 
Península Ibérica (López Medina, 2004: 119), situación en la que entendemos sería determinante no sólo la abrupta orografía del terreno, sino también la cantidad de agua disponible, localizándose inicialmente en espacios naturales como ríos, lagos, estanques o lagunas.

\subsection{Los textos epigráficos}

A los textos epigráficos no se les ha prestado demasiada atención a la hora de analizar los recursos hídricos y el beneficio que las comunidades locales hicieron de ellos en el sur peninsular, entre otras cuestiones, porque salvo las leyes municipales (que analizaremos en el siguiente apartado) no se han localizado documentos de extraordinaria relevancia, como ocurre en otras zonas de Hispania, especialmente en la provincia Tarraconense. Creemos sin embargo, que es un campo que todavía está por estudiar y que puede aportar importantes datos en relación con las cuestiones que aquí estamos tratando. En general son parcos en términos que aludan a estos recursos, pero sí nos sirven de ayuda para saber que estos lugares estuvieron explotados. Y es ahí, uniéndolos a la arqueología, cuando los datos que nos aportan pueden ser significativos a la hora de inferir un conocimiento más aproximado sobre su explotación.

Vamos a citar un ejemplo en el que estamos trabajando, la civitas de Singila Barba (Cjo. del Castillón, Antequera, Málaga) y su relación con la Laguna de Fuente Piedra. Se trata del humedal de mayores de dimensiones de la Comarca de Antequera, y está ubicado en una zona con especial relevancia de humedales, a lo que hay que unir el paso del río Guadalhorce y los manantiales. El municipio de Singila Barba presenta además unas tierras fértiles para la agricultura, que sin duda alguna se debieron beneficiar de los recursos hídricos de la Comarca, que formarían parte de los loca publica agrestia.

Pero volvamos al caso concreto de la Laguna de Fuente Piedra, ésta forma parte de una cuenca endorreica, que está alimentada por arroyos, entre los que destacan el de Santillán y el de Los Arenales ${ }^{49}$. Actualmente la precipitación media anual es de 460 $\mathrm{mm} / \mathrm{a}$, y se seca durante el estiaje, y en la cuenca se sitúan varias poblaciones que se abastecen de las aguas subterráneas mediante pozos, y cuya actividad económica preponderante es la agricultura. Se trata de una laguna cuyas aguas presentan una alta salinidad (10 veces más la del agua del mar), lo que está favorecida por la considerable evaporación.

Con respecto a la arqueología, los últimos análisis de poblamiento en torno a ella arrojan un total de 120 yacimientos que se pueden adscribir al periodo romano, y que se ubican principalmente junto a la laguna y Arroyo de Santillán ${ }^{50}$. Éstos se han vinculado especialmente a la explotación agrícola (principalmente para la producción de aceite), minera y de la sal ${ }^{51}$. Y algunos de ellos presentan epígrafes funerarios, en concreto CIL $\mathrm{II}^{2} / 5,834$ de Herriza, la CIL II ${ }^{2} / 5,833$ de Vicaría y CIL II'2/5, 832 de Fuente de

\footnotetext{
${ }^{49}$ Sobre la constitución geológica de esta zona, especialmente kárstica, precipitaciones, hidrogeología, etc. véase Heredia et al. (2009) y Linares (1990).

${ }^{50}$ Recio Ruiz (2007). Estos 120 yacimientos están catalogados en las pp. 542-544, sólo como romanos, sin precisar más su cronología, y posteriormente son identificados con asentamientos tipo villa vinculándolos al modelo de producción esclavista (p. 546). Sería necesario un estudio más detallado de ellos y su valoración con respecto a los materiales localizados y su extensión.

51 Corrales Aguilar (1997-1998: 92 y 94) y Recio (2007: 547). En cuanto a la explotación de la sal: Gozalbes Cravioto - Muñoz Hidalgo (1986).
} 
$\operatorname{Piedra}^{52}$, lo que confirman la importancia del poblamiento junto a la laguna. Con respecto a los dos primeros, su vinculación con asentamientos tipo villa está corroborada por los materiales que se encuentran en ellas, en concreto en el Cjo. de Herriza y en la Vicaría ${ }^{53}$, por lo que no es de extrañar la presencia de recintos dedicados a necrópolis, como probarían ambos epígrafes; posiblemente lo mismo debiéramos aplicar al tercero. Por último hallamos una cuarta inscripción, CIL $\mathrm{II}^{2} / 5,772$ de Peñuela ${ }^{54}$, en este caso se trata de una inscripción honorífica, que parece estar en una posición secundaria. Aunque ninguna hace alusión en concreto al aprovechamiento del agua, volvemos a insistir en su importancia, pues confirman los datos aportados por la arqueología en relación con el aprovechamiento de estos recursos.

\subsection{Los textos jurídicos}

La importancia de la gestión del agua en época romana tiene su expresión en la legislación que se produce durante esta época, tanto a nivel local, donde se pueden analizar las leyes municipales, como de carácter general, con ejemplos tan relevantes como los que aporta el Digesto o el Codex Theodosianus. En la Península Ibérica, en relación con la irrigación, son de especial relevancia los textos jurídicos de la Tarraconense, como la tabula Contrebiensis y la lex rivi Hiberiensis (Fatás, 1980; Beltrán, 2005, 2006 y 2011). En éstos, además, se pueden analizar las relaciones entre distintas civitates o comunidades y apreciar la importancia que alcanzaron los conflictos para gestionar y controlar el uso del agua asociado a la actividad agrícola.

En este apartado nos vamos a centrar en el análisis de los textos jurídicos de la Bética. Se trata de leyes de época tardorrepublicana y altoimperial, nos referimos a lex Ursonensis y la lex Irnitana ${ }^{55}$. En ellas, como ya hemos dicho, se muestra el interés de las autoridades locales por regular la captación y aprovechamiento del agua tanto para consumo humano como para actividades agrícolas, sin embargo no aportan información significativa para llevar a cabo una conceptualización de los humedales.

La primera está asociada a la colonia de Urso (Osuna, Sevilla) cuya deductio se realiza por César entre los años 47-44 a.C. (González, 1989). Es la que más datos nos proporciona en relación con la mencionada regulación y su posterior redistribución de estas aguas para riego. Posiblemente esta circunstancia se deba a la creación de una civitas de nueva planta, por lo que la reglamentación debería ser más detallada que en el caso de otras civitates con una estructura urbana previa. En este sentido, la lex coloniae Genitivae Iuliae sen Ursonensis se produce en un momento posterior a su fundación, en concreto por Marco Antonio, posiblemente tras el asesinato de César, y reglamenta el uso de las aguas de la

\footnotetext{
${ }^{52}$ CIL II $2 / 5$, 834: D(is) M(anibus) S(acrum) / THEMISON / AN(norum) XXXXIII / P(ius) I(n) S(uis) S(it) T(ibi) T(erra) L(enis). CIL II ${ }^{2} / 5$, 833: D(is) M(anibus) S(acrum) / FLAVI $<V>S$ SEX/TIO ANNO/RVM XXVIII / PIVS IN SVIS / S(it) T(ibi) T(erra) L(euis). CIL II²/5, 832: D(is) M(anibus) S(acrum) / Q(uintus) CORNELIVS SECV/NDINVS SINGIL(iensis) / ANN(orum) LXI PIVS IN SVIS / H(ic) S(itus) E(st) S(it) T(ibi) T(erra) L(euis)

53 Recio Ruiz (2007), sobre Cjo. de Herriza (n 26), 543 y 546; sobre Vicaría (n ${ }^{\circ}$ 6) 541 y 543 . Sobre la presencia de restos de molinos de tipo mola olearia en Vicaría: Corrales Aguilar (1997-1998: 92).

${ }^{54}$ CIL II ${ }^{2} / 5$, 772: M(arcus) CORNELIVS PRIMIGENIVS SING(iliensis) / OB BENEFICIVM QVOD AB ORDINE SING(iliensi) / LOCVM ACCEPERAM / IN QVO STATVAM PONEREM / M(arci) CORNELI SATVRNINI F(ilii) MEI / LVPAN CVM INFANTIBVS DVOBVS / D(edi) D(edicavi)

55 Para un análisis más detallado de estas leyes y su relación con el control y aprovechamiento del agua véase Cañizar (2009 y 2011).
} 
colonia en los capítulos 77, 79, 99, 100 y 104; en concreto el 99 y el 104 hacen referencia a las de riego y a los canales que delimitaban los campos ${ }^{56}$.

Con respecto a la segunda, la lex Imitana, o ley municipal de Irni (El Saucejo, Sevilla), datada en época flavia, tras el Edicto de Latinidad de Vespasiano, concretamente durante el reinado de Domiciano (81-96 d.C. $)^{57}$. Entre sus capítulos, dos de ellos hacen mención expresa a la gestión de las aguas y su conducción, nos referimos al 19 y 82 . Como podemos observar, la regulación en este caso es menor, pues se trata de un núcleo urbano autóctono ya existente antes de conseguir su promoción a municipio, pero que venía actuando como tal desde momentos anteriores, por lo que la población ya tendría un sistema de gestión de las aguas previo.

En primer lugar, nos interesa analizar qué tipo de aguas se consideran públicas. El Digesto (43.7.1) nos dice sobre lo público: "Debe permitirse a todo el mundo el uso general de lo que es público, como las vías y los caminos públicos (...)”; además sobre las aguas se afirma que el aqua profluens (Dig. 1.8.2.1) es de uso público, aunque en algunas ocasiones su apropiación pueda ser particular, según el carácter público o privado de la propiedad donde surge (Dig. 39.3.8). En este sentido cuando el agua es recogida por el dominus de un fundus mediante una conducción pasa a ser privada, si bien se puede establecer su uso público a través de la constitución de una servidumbre de acueducto (Rodríguez Neila, 1988, 230-231). Por la lex Ursonensis desconocemos qué aguas son las públicas, pero no cabe duda que en este carácter debían entrar las de los ríos cuyo caudal era constante (Dig. 43.12.1.3). Así en el cap. 79 de la lex Ursonensis, se hace alusión a la existencia de un servicio de aguas público anterior a la fundación de la colonia, donde se cita ríos, torrentes, fuentes, lagos, estanques y lagunas (Qui fluvi rivi fontes lacus aquae stagna paludes sunt in agro...). Esta situación previa a la fundación colonial es fundamental, pues así su carácter público se podría mantener tras el reparto del ager y a la asignación de propiedades privadas (Rodríguez Neila, 1988, 251).

Por otro lado, hay que destacar que dentro de este tipo de aguas se encuentran las que se designan por los términos lacus (lago), stagna (estanques) y paludes (lagunas o pantanos), a los que se puede asociar el de fossa (presa). Así en el Digesto (43.14.1.3-5) se les define como "Lago es el que tiene agua permanentemente. Estanque es el que tiene temporalmente agua que se reserva en él, la cual suele recogerse allí en el invierno. Presa es un receptáculo construido". En cuanto a los lagos, aquellos de gran extensión y con agua constante reciben el mismo tratamiento que los ríos (Dig. 8.2.28), pero no así aquellos como los estanques. Sin embargo, como vemos mediante la lex Ursonensis (cap. 79), se decreta la existencia de estanques y lagunas de uso público, lo que está apoyado por el Digesto (43.14.6). Aquí se indica que algunos de éstos deberían poseer carácter público, por lo que éste dependía de la propiedad en la que se encontraran las aguas (Cañizar, 2009). En el caso concreto de Urso su uso público también se puede deber a la pervivencia de ordenamientos previos a la deductio de la colonia. De todo ello se puede deducir que el uso del agua de carácter público estaba fuertemente regulado.

Por otro lado, ¿quiénes son las personas que están al cargo de las obras de construcción y reparación de canales, es decir, de la supervisión y regularización de la distribución y uso de las aguas? Sobre la reparación de canales, se ve la importancia para el consumo

\footnotetext{
${ }^{56}$ Sobre la deductio de la colonia y posterior promulgación de su lex véase D’Ors (1997). Sobre la lex Ursonensis véase D’Ors (1953) y González Fernández (1990).

57 Traducción de D’Ors y D’Ors (1989).
} 
humano: "ya que si éstas no se reparan, se pierde todo uso del agua y podría la gente morir de sed, porque es claro que el agua no puede llegar si no se repara la acequia, en tanto, cuando no se repara el camino, tan sólo hay dificultad para pasar y llevar el ganado" (Dig. 43.21.4). En este sentido en los capítulos 99 y 104 de la lex Ursonensis, se establece que estas obras debían ser competencia de los duunviros, máximo cargo administrativo de la colonia; lo que está refrendado por el cap. 77 que establece que los principales magistrados, ediles y duunviros están al cuidado de las obras públicas, entre ellas cloacas y acequias (Si quis vias fossas cloacas...), y por la lex Irnitana en sus capítulos 19 Rúbrica y 82 Rúbrica.

En este sentido, también es relevante el cap. 99 de la lex Ursonensis que trata la expropiación forzosa, sin indemnizaciones, de propiedades a particulares para construir conducciones de agua de carácter público, si los duunviros lo estiman necesario. Para ello la decisión debía ser aprobada por la mayoría del senatus o curia, en concreto dos tercios de los decuriones. Este elevado porcentaje del voto de los decuriones que se requería para llevar a cabo la expropiación, se supone que sería para perjudicar lo menos posible a los particulares; de hecho esta regulación no podía afectar a los edificios ya construidos (González Román, 2011). En este sentido debemos tener en cuenta que los miembros de la curia pertenecen a las elites locales, y que son éstas las grandes propietarias, por lo que podemos ver en este capítulo una forma de legislar a favor de sus propios intereses.

De lo anterior, se deduce la importancia de la construcción de acequias, que se relacionan con la irrigación, pero ¿qué aguas podrían estar vinculadas con ésta? Obviamente se podrían utilizar todas aquellas de propiedades particulares, las sometidas a servidumbre de acueducto, y un tipo, que destacamos, el aqua caduca. En este sentido, es relevante el cap. 100 de la lex Ursonensis, que establece la concesión del aprovechamiento por parte de los colonos de la civitas del aqua caduca, es decir, aquella que se derramaba de los depósitos o se filtraba de las tuberías (Fron., De aquaeductu, 94, 107, 109, 110; Rodríguez Neila, 1988). Podemos suponer que ésta fue utilizada para el riego entre otros usos (alfares, termas, fullonicae...), y que debió tener un especial tratamiento en aquellas comunidades donde la captación de este tipo de aguas aseguraba el cultivo de determinados productos. Para aprovecharla lo tenían que solicitar a los duunviros y necesitaba la aprobación de 40 decuriones del senado local, por lo que era ilegal su apropiación indebida. En esta ocasión se hace mención expresa a evitar posibles perjuicios a particulares (... sine privati iniuria).

Para finalizar, podemos deducir del cap. 104 de la lex Ursonensis que el agua usada para la irrigación estaba regulada, pues en éste se alude a la multa que se debía pagar al obturar o interceptar las acequias dedicadas al riego, en total ésta se establecía en mil sestercios.

Si tenemos en cuenta que todos los municipios hispanos creados a partir del edicto de Vespasiano, y por lo tanto los béticos, debieron tener una lex municipalis, concreta e individualizada (Mentxaka, 1993 y Muñiz Coello, 1984-1985), debió existir una fase intermedia entre el edictum y la ejecución de las distintas leges municipales, en la cual los municipios se fueron adaptando al nuevo modo de vida administrativo. Para algunos autores, esta ley tendría como base una ley general flavia, llamada lex Flavia Municipalis, debido a la uniformidad que se observa al analizar leyes como la de Irni, Malaca, Salpensa, etc. Sin entrar en el debate de la existencia de ésta, debemos reconocer que lo que hemos visto en este apartado sobre la gestión de las aguas fue similar en todas las comunidades de la Bética. 


\section{Conclusiones}

Pese a su escasez y no haber sido explotadas plenamente, las fuentes literarias, epigráficas y jurídicas nos aportan una valiosa base informativa que puede completar el estudio arqueológico del medio en el que se inserta la red de riegos. Ciertamente quedan interrogantes sin resolver en torno al regadío y la explotación de los humedales de la Bética y hay otras cuestiones que tendrán que revisarse en el futuro, pero hemos de esperar a que los cada vez más frecuentes estudios específicos se encuentren más avanzados y se cubran campos a los que hasta ahora se había dedicado poca atención por resultar marginales o poco espectaculares, como son las zonas anegadas y los ámbitos rurales.

De otro lado, el aprovechamiento de las aguas, tanto para consumo humano como para su utilización en actividades productivas como la irrigación, es de capital importancia para las comunidades de la Bética. En especial nos llama la atención la relevancia que debió tener el control de los humedales, pues como hemos destacado, esta provincia está formada por una gran diversidad de espacios naturales con recursos acuíferos muy diversos. Todo ello nos indica que para sociedades como la romana el control y aprovechamiento del agua, incluida la de riego, no fue en modo alguno una cuestión baladí.

\section{Referencias}

Alonso Núñez, J.M. (1999): La Turdetania de Estrabón, en G. Cruz Andreotti, Coord., Estrabón e Iberia: Nuevas perspectivas de estudio, Málaga, pp. 101-119.

Arce, J. (1989): Estrabón sobre la Bética, en J. González, Ed., Estudios sobre Urso. Colonia Iulia Genetiva, Sevilla, pp. 213-222.

Arteaga, O. et alii (1985): Investigaciones geológicas y arqueológicas sobre los cambios de la línea de costa en el litoral de la Andalucía Mediterránea. Informe preliminar (1985), Anuario Arqueológico de Andalucía II: 117-122.

Arrayás Morales, I. - López Medina, Mã. (2009): Paysage et climat de la péninsule ibérique pendant l'Antiquité. Quelques exemples: la côte centrale catalane et le littoral almérien, en E. Hermon, Dir., Societé et climats dans l'Empire romain. Perspective historique et systémique de la gestion des ressources en eau dans l'Empire romain, Napoli, pp. 281-308.

Beltrán Lloris, F. (2005): Nuevas perspectivas sobre el riego en Hispania: la Lex riui Hiberiensis, en L. Hernández Guerra, Ed., Actas del II Congreso Internacional de Historia Antigua: La Hispania de los Antoninos (98-180), Valladolid, pp. 129-139.

Beltrán Lloris, F. (2006): An irrigation Decree from Roman Spain: The Lex Rivi Hiberiensis, JRS, 96: 147-197. 
Beltrán Lloris, F. (2011): El agua y las relaciones intercomunitarias en la Tarraconense, en L. Lágostena - J.L. Cañizar - L. Pons, Eds., Aquam perducendam curavit. Captación, uso y administración del agua en las ciudades de la Bética y el occidente romano, Cádiz, pp. 21-40.

Beltrán Lloris, F.; Willi, A. (2011): El regadío en la Hispania romana. Estado de la cuestión, Cuadernos de Prehistoria y Arqueología de la Universidad de Granada, 21: 9-56.

Bianco, A.D. (2007): Aqua ducta, aqua distributa. La gestione delle risorse idriche in età imperiale, Torino.

Brunn, C. (2012): Roman emperors and legislation on public water use in the Roman Empire: clarifications and problems, $W$ ater History, 4/1: 11-33.

Callebat, L. (1973): Vitruve, De l'architecture, Livre VIII, Les Belles Lettres, Paris.

Cañizar Palacios, J.L. (2011): El agua en la escritura pública del poder: panegíricos, legislación imperial y leyes municipales, en L. Lágostena - J.L. Cañizar - L. Pons, Eds., Aquam perducendam curavit. Captación, uso y administración del agua en las ciudades de la Bética y el occidente romano, Cádiz, pp. 449-460.

Cañizar Palacios, J.L. (2009): Fuentes jurídicas sobre la gestión y administración del agua: el espacio gaditano, en L.G. Lagostena Barrios - F.B. Zuleta Alejandro, Coords., La captación, los usos y la administración del agua en Baetica: Estudios sobre el abastecimiento bídrico en comunidades civicas del Conventus Gaditanus, Cádiz, pp. 41-59.

Carrilero Millán, M. - López Medina, MãJ. - García Pardo, M. (2004): El paisaje del Sureste peninsular. Proceso histórico de cambio medioambiental iniciado en la Antigüedad, en J. Peñas - L. Gutiérrez, Eds., Biología de la conservación. Reflexiones, propuestas y estudios del Sureste Ibérico, Almería, pp. 39-58.

Castillo Pascual, Mã. (1996): Espacio en orden: el modelo gromático-romano de ordenación del territorio, La Rioja.

Corrales Aguilar, P. (1997-1998): Actividades económicas en la Comarca de Antequera (Málaga) en época romana, Mainake, XIX-XX: 89-105.

Cruz Andreotti, G. (2007): Acerca de Estrabón y la Turdetania-Bética, en G. Cruz Andreotti - P. Le Roux - P. Moret, Eds., La invención de una geografía de la Península Ibérica. II. La época imperial, Málaga pp. 251-270.

D’Ors, X. (1997): Observaciones formales sobre la composición de la ley Ursonense, Studia Historica. Historia Antigua, 15: 63-93 (86).

D’Ors, A. (1953): Epigrafía jurídica de la España romana, Madrid.

D’Ors, A. y D’Ors, J. (1989): Lex Irnitana (Texto bilingüe), Santiago de Compostela.

Fatás, G. (1980): Contrebia Belaisca (Botorrita, Zaragoza). II. Tabula Contrebiensis, Zaragoza. 
Fornell Muñoz, A. (2001): La villa canónica según los agrónomos latinos y su constatación arqueológica en Andalucía, Humanitas, 1: 1-19.

Fornell Muñoz, A. (2005): Las villae romanas en la Andalucía mediterránea y del Estrecho, Jaén

Fornell Muñoz, A. (2011): Control y uso del agua en las villas de la Bética, en L. Lágostena-J.L. Cañizar-L. Pons, Eds., Aquam perducendam curavit. Captación, uso y administración del agua en las ciudades de la Bética y el occidente romano, Cádiz, pp. 365-381.

Gómez Espelosín, J. (2007): Estrabón, Geografía de Iberia, Clásicos de Grecia y Roma, Alianza Editorial, Madrid, p. 144.

González Fernández, J. (1990): Bronces jurídicos romanos de Andalucia, Sevilla, pp. 33-36; CIL II2/5, 1022.

González Román, C. (2011): El agua en las ciudades de la Bética: organización y funciones, en L. Lágostena - J.L. Cañizar - L. Pons, Eds., Aquam perducendam curavit. Captación, uso y administración del agua en las ciudades de la Bética y el occidente romano, Cádiz, pp. 41-65.

Goold, G.P. Ed. (1979): Pliny, Natural History, The Loeb Classical Library, Harvard University Press

Gozalbes Cravioto, C. - Muñoz Hidalgo, F. (1986): Fuente de Piedra: la vía romana de la sal, Jábega 53: 20-23.

Hermon, E., Ed., (2010): Riparia dans l'Empire romain. Pour la définition du concept, BAR International Series 2066, Oxford.

Hermon, E. - Watelet, A., Dirs. (2014): Riparia, un patrimoine culturel. La gestión intégrée des bords de l'eau, BAR International Series 2587, Oxford.

Hernando Gonzalo, A. (1987): ¿Evolución cultural diferencial del Calcolítico entre las zonas áridas y húmedas del sureste español?, Trabajos de Prehistoria, 44: 171-200.

Heredia, J. et al. (2009): Caracterización de un sistema hidrogeológico complejo con variabilidad extrema de la densidad: Laguna de Fuente de Piedra (Málaga). Vinculación con un sistema kárstico regional hipersalino, Boletín Geológico y Minero, 120/3: 423-442

Hoffmann, G. (1988): Holozänstratigraphie und Küstenlinienverlagerung an der Andalusischen Mittelmeerküste, Berichte aus dem Fachbereich Geowissenschaften der Universität Bremen 2, Bremen.

Holgado Redondo, A., Ed., (1988): De los trabajos del campo. Lucio Junio Moderato Columela, Siglo XXI, Madrid, XV-XVI. 
Lagóstena Barrios, L.G. - Cañizar Palacios, J.L. (2010): Salinae maritimae en Baetica: condiciones ambientales, consideración jurídica y relaciones intercomunitarias, en E. Hermon, Ed., op.cit., pp. 67-84.

Leone, A. (2012): Water management in late antique North Africa: agricultural irrigation, Water History, 4/1: 119-133.

Leveau, Ph. - Paillet, J.-L. (1983): Alimentation en eau et développement urbain à Caesarea de Maurétanie, en J.-P. Boucher, Ed., Actes des Journées d'Études sur les aqueducs romains. Tagung über römische Wasserversorgungsanlagen, Lyon 26-28 mai 1977, Paris, pp. 231-234

Linares, L. (1990): Hidrogeología de la laguna de Fuente de Piedra (Málaga), Granada.

López Medina, M J. (1996): Las civitates del Sureste Peninsular durante el Alto Imperio. Algunas cuestiones sobre su urbanismo y su territorio, Florentia Iliberritana, 7: 171-185.

López Medina, Maj. (1998-1999): Algunas cuestiones sobre: "El agua en el sureste peninsular durante época romana. Su aprovechamiento para la agricultura", Lucentum, XVII-XVIII: 243-253.

López Medina, Mã J (2000): Algunas cuestiones sobre la familia campesina en el Alto Imperio: El ejemplo del sureste peninsular, Gerión, 18: 357-393.

López Medina, Mã. (2004): Ciudad y territorio en el sureste peninsular durante época romana, Ediciones Clásicas, Madrid.

López Medina, Maj. (2008): El trabajo de las mujeres en la unidades domésticas campesinas del Sureste peninsular durante el Alto Imperio, en T. Escoriza Mateu - $\mathrm{M}^{\mathrm{a}} \mathrm{J}$. López Medina - A. Navarro Ortega, Eds., Mujeres y Arqueología. Nuevas aportaciones desde el materialismo histórico. Homenaje al Profesor Manuel Carrilero Millán, Granada, pp. 199-250.

López Medina, Mã. - Carrilero Millán, M. (2000): Reconstrucción paleoambiental del Sureste peninsular en la Antigüedad, en T. Naco et alii, Eds., Anàlisis paleoambientals $i$ estudi del territorio, Barcelona, pp. 49-65.

Maganzani, L. (2014): Le réglement des riparia dans les communautés d'irrigation dans le monde romain, en E. Hermon - A. Watelet, Dirs., Riparia, un patrimoine culturel. La gestión intégrée des bords de l'eau, pp. 225-231.

McMaster, A. (2014): Un lieu de verdure et d'agrément: espaces riverains et loci amoeni dans la poésie la tine, en E. Hermon - A. Watelet, Dirs., Riparia, un patrimoine culturel. La gestion intégrée des bords de t'eau, pp. 217-224.

Mentxaka, R. (1993): El Senado municipal de la Bética bispana a la luz de la Lex Irnitana, Vitoria, p. 51.

Moreno Herrero, S. (2012): El Emperador y los ríos. Religión, ingeniería y política en el Imperio Romano, UNED, Madrid, pp. 91-125.

Moreno Soldevila, R. (2003): Water, Desire and the Elusive Nature of Martial IV 22, Exemplaria, 7: 149-163. 
Muñiz Coello, J. (1984-1985): La política municipal de los flavios en Hispania. El municipium Irnitanum, Studia Historica, II-III/1: 151-176 (en especial pp. 154-155).

Pantaleón-Cano, J. et alii (1996): Dinámica del paisaje vegetal durante el Neolítico en la vertiente mediterránea de la Península Ibérica e Islas Baleares, Rubricatum, 1-2: 29-34

Parroni, P.G. (1965): Vibius Sequestris de fluminibus fontibus lacubus etc., MilanoVarese

Parroni, P. (1984): Pomponii Melae, De Chorographia. Libri Tres, Roma.

Parroni, P. (2007): La Spagna di Pomponio Mela, en G. Cruz Andreotti - P. Le Roux P. Moret, Eds., La invención de una geografía de la Península Ibérica. II. La época imperial, Málaga, pp. 81-93.

Prieto Arciniega, A. - Arrayás Morales, I. - López Medina, Mả. (2011): Sobre los sistemas de regadío en época romana. El caso del territorio de Tarragona y Almería, en L. Lágostena - J.L. Cañizar - L. Pons, Eds., Aquam perducendam curavit. Captación, uso y administración del agua en las ciudades de la Bética y el occidente romano, Cádiz, pp. 383-396.

Perotti, P. (2004): Note a Vibio Sequestre, Giornale Italiano di Filologia, 56/1, 87-99.

Peyras, J. (1995): Écrits d'arpentage et hauts fonctionnaires équestres de l'Antiquité Tardive. I, L'Afrique du Nord, DHA, 21/2: 149-204

Peyras, J. (2010): Les riparia dans l'Afrique Proconsulaire, en E. Hermon, Ed., op.cit., pp. 113-130.

Peyras, J. (2014): Les riparia dans les écrits gromatiques, en E. Hermon - A. Watelet, Dirs., op.cit., pp. 243-254 (en especial pp. 245-246).

Recio Ruiz, A. (2007): Fuente de Piedra (Málaga) y el Cerro de la Virgen. Bases arqueológicas y expolio, Mainake, XXIX: 541-556.

Rodríguez Neila, J.F. (1988): Aqua pública y política municipal romana, Gerión, 6: 223252.

Shaw, B.D. (1982): Lamasba: an ancient irrigation community, Antiquités africaines, 18: 61-103.

Sáez Fernández, P. (1990): Estudio sobre una inscripción catastral colindante con Lacimurga, Habis, 21: 205-227.

Serrano Peña, J.L. (2004): Aurgi. Estudio del municipio romano desde la arqueología urbana de Jaén 1985-1995, Jaén pp. 25-71.

Serrano Peña, J.L. et alii (2002): La paleomorfología de Marroquíes Bajos. Primeras propuestas, Arqueología y Territorio Medieval, 9: 7-36.

Tovar, A. (1975): Columela y el vino de Jerez, en Homenaje Nacional a Lucio Junio Moderato Columela, Cádiz, pp. 93-99. 
Zafra Sánchez, J. (1997): Estructuras hidráulicas romanas e islámicas junto al arroyo A de Marroquíes Bajos (Jaén), Arqueología y Territorio Medieval, 14: 112-113. 